\title{
New traffic light on Th17 Avenue
}

\author{
Cell Research (2018) 28:139-140. doi:10.1038/cr.2017.156; published online 8 December 2017
}

\begin{abstract}
Activation of STAT3-coupled receptors along with TGF- $\beta$ signaling are fundamental for Th17 cell differentiation both in vivo and in vitro. A recent paper shows that TGF- $\beta$ signaling relieves SKI-mediated transcriptional repression of Rorc, the key regulator of the Th17 program.
\end{abstract}

Thelper 17 (Th17) cells are abundant at mucosal surfaces, where they are induced by commensal microbiota and produce the cytokines IL-17A, IL-17F and IL-22. These cytokines are critical for maintenance of mucosal homeostasis through clearance of extracellular bacterial and fungal pathogens. However, unharnessed Th17 responses in human and mouse contribute to autoimmune diseases, including psoriasis, Crohn's disease, arthritis, and multiple sclerosis. Differentiation of Th17 cells from naïve $\mathrm{CD}^{+} \mathrm{T}$ cells is dependent on their expression of the nuclear receptor ROR $\gamma t$, which regulates the transcription programs necessary for Th17 cell-mediated homeostasis and pathogenesis [1].

In vitro differentiation of Th 17 cells can be achieved with a combination of IL- 6 and TGF- $\beta$, but IL- 23 and IL- $1 \beta$ are additionally required for Th17-mediated pathogenicity in mouse models of autoimmunity [2,3]. It has been argued that TGF- $\beta$ is dispensable under some conditions both in vitro and in vivo and, indeed, Th17 cells can be differentiated in vitro by a combination of IL-6, IL-23 and IL-1 $\beta$ [4]. IL-6, IL-21 and IL-23 induce Th17 cell differentiation through the STAT3 signaling pathway, which has at least two functions: induction of ROR $\gamma$ t expression and down-regulation of TGF- $\beta$-induced Foxp3, the key tran- scription factor for regulatory $\mathrm{T}$ cells (Tregs) [5]. However, the precise function of TGF- $\beta$ has remained obscure, as it induces little expression of ROR $\gamma \mathrm{t}$ or cytokines. Earlier papers from two groups shed some light on the function of TGF- $\beta$, showing that IL- 6 alone could induce dramatic IL-17A/F expression in STAT6/T-bet double knockout T cells in the absence of TGF- $\beta[6,7]$. Moreover, administration of anti-IL-6 antibody, but not anti-TGF- $\beta$ antibody, blocked Th17-mediated autoimmune diseases in BALB/c mice deficient for STAT6 and T-bet. Both studies suggested that TGF- $\beta$ promotes Th17 programming by suppressing Th1 and Th2 cell differentiation upon TCR signaling [6, 7]. Due to the genetic background difference, it is not clear whether TGF- $\beta$ has a similar function in C57BL/6 mice.

TGF- $\beta$ binds to its cognate receptor complex composed of T $\beta$ RI and T $\beta$ RII and triggers T $\beta R I$ kinase activity to phosphorylate SMAD2 and SMAD3. Phosphorylated SMAD2/SMAD3 then form a complex with SMAD4 to translocate into the nucleus to modulate gene expression. Single SMAD protein loss causes impairment of induced Treg (iTreg) but not thymus-derived Treg cell differentiation. However, whether single SMAD protein loss contributes to Th17 differentiation is controversial.

Wan and colleagues previously reported that Smad4 deletion in $\mathrm{CD} 4^{+} \mathrm{T}$ cells rescued lethal Th1 autoimmunity in T $\beta$ RII-deficient mice by limiting proliferation of the mutant T cells [8]. In their new paper published in Nature, they describe in vitro differentiation of SMAD4-deficient T cells into Th17 cells in response to IL-6 in the absence of TGF- $\beta$ signaling [9]. They also show that $\mathrm{T}$ cells deficient for both SMAD4 and TRRII were as competent as wildtype $\mathrm{T}$ cells to differentiate into Th17 cells at steady state conditions in vivo and to mediate Th17 cell-dependent experimental autoimmune encephalomyelitis. These results suggest that SMAD4-deficient T cells could differentiate into both pathogenic and non-pathogenic Th17 cells without TGF- $\beta$ signaling in vivo. To investigate a molecular mechanism for this surprising finding, they examined transcription of Th17 cell genes in the absence of SMAD4, and found early expression of ROR $\gamma \mathrm{t}$ in response to IL-6 alone in the mutant T cells. Moreover, SMAD4 specifically bound to the Rorc locus but not $I L 17 a$ or $I L 17 f$ loci, suggesting that it directly regulated ROR $\gamma t$ expression. Since SMAD4 remained bound to the Rorc locus even in the presence of TGF- $\beta$, the authors reasoned that it might form different complexes to regulate $\mathrm{ROR} \gamma \mathrm{t}$ expression in the presence or absence of TGF- $\beta$ signaling. Using a proteomics strategy, they then identified SKI as a SMAD4 partner in the absence, but not in the presence, of TGF- $\beta$ signaling. SKI, a component of a histone deacetylase complex, was previously reported as a negative regulator of TGF- $\beta$ signaling through its direct interaction with SMAD proteins [10]. Thus, in the absence of TGF- $\beta$, the recruitment of SKI by SMAD4 resulted in the deacetylation of Lys9 in histone H3 (H3K9) and repression of Rorc transcription. Upon addition of TGF- $\beta$, degradation of SKI protein occurred even when protein synthesis was inhibited, allowing for STAT3-dependent H3K9 


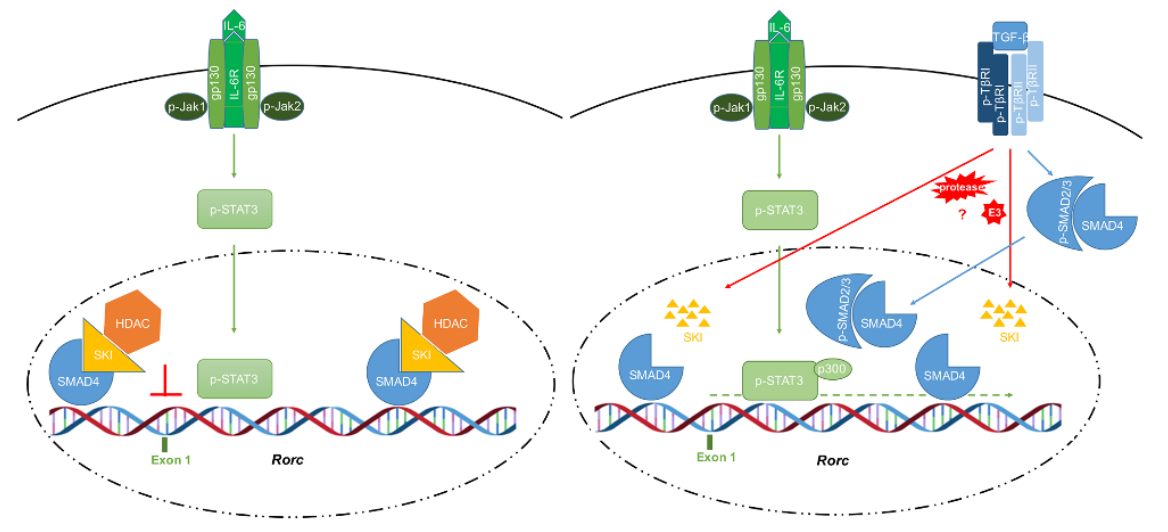

Figure 1 Schematic diagrams of TGF- $\beta$ signaling function in Th17 cell differentiation. Without TGF- $\beta$ signaling (left panel), SMAD4 migrates into nucleus, binds to multiple specific sites in the Rorc gene, and recruits a complex of SKI and H3K9 histone deacetylase that suppresses ROR $\gamma$ t expression. In response to TGF- $\beta$ stimuli (right panel), SKI is degraded by an unknown mechanism, allowing for H3K9 acetylation in the Rorc locus and induction of ROR $\gamma$ t expression in response to phosphorylated STAT3 (p-STAT3) and other transcriptional regulators.

acetylation at the Rorc locus, ROR $\gamma t$ upregulation and Th17 cell differentiation. In an elegant series of experiments, the authors then showed that a direct interaction between SKI and SMAD4 was required for the suppressive function of the complex (Figure 1). Consistent with their results, overexpression of SKI suppressed $\mathrm{H} 3 \mathrm{~K} 9$ acetylation at the Rorc locus, whereas SKI deletion, like that of SMAD4, promoted ROR $\gamma \mathrm{t}$ expression in response to IL-6 signaling. The mechanism of SKI degradation following TGF- $\beta$ receptor signaling was not explored, although there was at least partial dependency on expression of SMAD2 and SMAD3.

The above findings by Wan's group provide much needed detail as to the mechanism by which TGF- $\beta$ regulates Th17 cell differentiation. In contrast to its role in iTreg cell differentiation, in which it is thought to directly induce transcription, the TGF- $\beta$ signaling pathway in Th17 cells appears to involve de-repression of the Rorc locus, by inducing the degradation of the SMAD4-bound SKI histone deacetylation complex. It remains possible, however, that TGF- $\beta$ receptor signaling and SMAD phosphorylation also par- ticipate in regulation of complexes that activate other genes in the Th17 differentiation program. The new results offer potential mechanisms to explain the findings of TGF- $\beta$-independent Th17 cell differentiation [4]. For example, some cytokines other than TGF- $\beta$ may similarly disrupt SMAD4-dependent corepressor complex binding to Rorc. There are multiple additional mechanistic questions that can now be addressed: 1) Is the SMAD4-SKI complex the only repressor at the Rorc locus that can be reversed following TGF- $\beta$ signaling? For example, STAT6/T-bet double knockout $\mathrm{T}$ cells also differentiated into Th17 cells in response to IL-6 in the absence of TGF- $\beta$ signaling, but it is not known whether this required de-repression of the SMAD4 complex. This may indeed be the case, since RNA-seq data in the current paper show that SMAD4-knockout $\mathrm{T}$ cells cultured with IL-6 plus TGF $\beta$ R inhibitor had decreased expression of Th1/Th2-related genes, including Stat6, Gata3, Tbx21, Il12rb1, Ifng and Ifngr, consistent with the possibility that repression of the alternative differentiation pathways favors disruption of the SMAD4-SKI complex at the Rorc locus. 2) Is the
SKI/SMAD4 complex induced by IL-6 and STAT3-dependent signaling or is it constitutively bound at the Rorc locus and, if so, is repression established early in T cell development? Are the histones in the Rorc locus acetylated after SKI degradation or do they remain deacetylated until there is a separate induction signal (e.g., pSTAT3 binding)? 3) How is SKI degraded upon TGF- $\beta$ stimulation? Is there a specific E3 ligase inducing ubiquitin-mediated degradation or is there a specific protease that targets SKI? 4) Finally, why do T cells deficient for both SMAD4 and TBRII maintain pathogenicity in Th17 cell-mediated autoimmunity while Th1 autoimmunity is impaired? Further mechanistic understanding of how TGF- $\beta$ controls the balance of effector $\mathrm{T}$ cells will likely facilitate the development of better targeted therapeutics for inflammatory diseases and immune-oncology.

\section{Hao $\mathrm{Xu}^{1}$, Dan R Littman ${ }^{1,2}$}

${ }^{1}$ Molecular Pathogenesis Program, The Kimmel Center for Biology and Medicine of the Skirball Institute, New York University School of Medicine, New York, NY 10016, USA; ${ }^{2}$ The Howard Hughes Medical Institute, USA

Correspondence: Dan R Littman

E-mail: Dan.Littman@med.nyu.edu

\section{References}

1 Ivanov II, McKenzie BS, Zhou L, et al. Cell 2006; 126:1121-1133.

2 Veldhoen M, Hocking RJ, Atkins CJ, et al. Immunity 2006; 24:179-189 .

3 Gaffen SL, Jain R, Garg AV, et al. Nat Rev Immunol 2014; 14:585-600.

4 Ghoreschi K, Laurence A, Yang XP, et al. Nature 2010; 467:967-971.

5 Korn T, Bettelli E, Oukka M, et al. Annu Rev Immunol 2009; 27:485-517.

6 Yang Y, Xu J, Niu Y, et al. J Immunol 2008; 181:8700-8710.

7 Das J, Ren G, Zhang L, et al. J Exp Med 2009; 206:2407-2416.

$8 \mathrm{Gu} \mathrm{AD}$, Zhang $\mathrm{S}$, Wang $\mathrm{Y}$, et al. Immunity 2015; 42:68-79.

9 Zhang S, Takaku M, Zou L, et al. Nature 2017; 551:105-109.

10 Liu X, Sun Y, Weinberg RA, et al. Cytokine Growth Factor Rev 2001; 12:1-8. 